\title{
Isoform D of vascular endothelial growth factor in systemic capillary leak syndrome: a case report
}

Soushi Ibata, Tsutomu Sato, Kohichi Takada, Ayumi Tatekoshi, Akari Hashimoto, Yusuke Kamihara, Wataru Jomen, Hiroto Horiguchi, Kaoru Ono, Kazuyuki Murase, Satoshi lyama, Koji Miyanishi, Yasushi Sato, Rishu Takimoto, Masayoshi Kobune and Junji Kato*

\begin{abstract}
Background: Systemic capillary leak syndrome is a rare condition characterized by episodic attacks of hypovolemia due to systemic capillary hyperpermeability, which results in profound hypotension and edema. Although the implication of vascular endothelial growth factor, angiopoietin-2, and C-X-C motif chemokine 10 has been suggested, the pathogenesis of systemic capillary leak syndrome remains unclear. In this report, we describe a case of systemic capillary leak syndrome in which serum isoform $D$ of vascular endothelial growth factor was elevated. To the best of our knowledge, this is the first reported case of systemic capillary leak syndrome in which isoform D of vascular endothelial growth factor is suggested as the plausible biomarker.
\end{abstract}

Case presentation: A 41-year-old Japanese man was transferred to our emergency department. He was hypotensive, tachycardic, and edematous over the trunk and all four limbs. He received aggressive intravenous fluid therapy and underwent fasciotomy of the right forearm to prevent muscle necrosis. A diagnosis of systemic capillary leak syndrome was suspected. The presence of serum monoclonal immunoglobulin $\mathrm{G}$ and $\mathrm{k}$ light chain supported this diagnosis. Prevention of hypotensive crises was unsuccessfully attempted with theophylline, intravenous immunoglobulin, high-dose dexamethasone, bortezomib, melphalan, and prednisolone; however, the patient's attacks dramatically disappeared after the introduction of thalidomide. The serum of the patient was stored soon after the onset of hypotensive crisis and analyzed to profile possible mediators responsible for the capillary leak. The concentration of vascular endothelial growth factor, angiopoietin-2, and C-X-C motif chemokine 10 were all within normal ranges. Meanwhile, we found that isoform D of vascular endothelial growth factor was elevated, which was normalized after the introduction of thalidomide.

Conclusions: In our patient, isoform D of vascular endothelial growth factor (instead of vascular endothelial growth factor) may have been a causative factor of hypotensive crises, since isoform D contributes to vascular endothelial growth factor receptor-2 signaling, which is the major mediator of the permeability-enhancing effects of vascular endothelial growth factor. We suggest the measurement of isoform $D$ of vascular endothelial growth factor in patients with systemic capillary leak syndrome in whose serum vascular endothelial growth factor is not elevated.

\footnotetext{
* Correspondence: jkato@sapmed.ac.jp

Department of Medical Oncology and Hematology, School of Medicine,

Sapporo Medical University, South-1, West-16, Chuo-ku, Sapporo, Japan
} 


\section{Background}

Systemic capillary leak syndrome (SCLS) is a rare condition characterized by episodic attacks of hypovolemia due to systemic capillary hyperpermeability, which results in profound hypotension and edema. Acute attacks of SCLS usually develop within a few hours and resolve within 1-4 days. The stereotypic laboratory abnormalities during acute attack are hemoconcentration (elevated hematocrit) and hypoalbuminemia. The presence of serum $M$ protein during the quiescent phase between the attacks is a notable common feature. Complications such as acute renal failure, rhabdomyolysis, arrhythmia, pericardial effusion, pleural effusion, deep venous thrombosis, and ischemic stroke during the attacks have been reported [1, 2]. These complications can be directly related to death, and reported survival rates are $89 \%$ at 1 year and $73 \%$ at 5 years [1]. Although the implication of vascular endothelial growth factor (VEGF), angiopoietin-2 (Ang-2), and C-X-C motif chemokine 10 (CXCL10) has been suggested [3-6], the pathogenesis of SCLS remains unclear.

Many agents have been tried sporadically with varying degrees of effectiveness, including corticosteroids, intravenous immunoglobulin (IVIG), antihistamines, plasmapheresis, and $\beta_{2}$-agonists (aminophylline, theophylline, or terbutaline) [2]. The anti-VEGF monoclonal antibody bevacizumab has been used successfully in one patient [7]; however, the same anti-VEGF therapy was used in another patient without success [3]. Thalidomide that targets an abnormal plasma cell population or its secreted products has been used in some patients, with improvement in symptoms achieved $[1,8]$.

In this report, we present a case of a man with SCLS refractory to corticosteroids, theophylline, IVIG, bortezomib, melphalan, and prednisolone, whose attacks were dramatically prevented by the introduction of thalidomide. We also demonstrate the elevated level of isoform D of VEGF (VEGF-D) in this patient's serum soon after the hypotensive crisis. To the best of our knowledge, this is the first reported case of SCLS in which VEGF-D is suggested as the plausible biomarker.

\section{Case presentation}

A 41-year-old Japanese man was transferred to our emergency department in October 2012 with complaints of headache and nausea. He was hypotensive (98/57 $\mathrm{mmHg})$, tachycardic (120-140 beats/minute), and edematous over the trunk and all four limbs. He had experienced the same episode 9 months previously. That first attack had been complicated by stroke, which caused right hemiplegia. Laboratory studies demonstrated elevated levels of hemoglobin $(18.6 \mathrm{~g} / \mathrm{dl})$ and hematocrit $(53.5 \%)$, as well as hypoalbuminemia $(2.2 \mathrm{~g} / \mathrm{dl})$. To keep his systolic blood pressure from dropping below $60 \mathrm{mmHg}$, he was given aggressive intravenous fluid therapy (500-1000 ml/hour). Following hydration for more than 12 hours, he complained of severe pain in his right forearm. His forearm compartments were tense and swollen. He underwent fasciotomy of the right forearm to prevent muscle necrosis. A volar longitudinal incision was made. The patient's hypotension lasted for 2 days, and his symptoms reversed quickly with massive diuresis. One week after fasciotomy, the wound was closed.

In view of these clinical features, a diagnosis of SCLS was suspected. Serum monoclonal immunoglobulin G (IgG) and $\mathrm{k}$ light chain was detected by immunofixation electrophoresis, supporting this diagnosis. The patient's IgG level was $1143 \mathrm{mg} / \mathrm{dl}$. A bone marrow study revealed $1.0 \%$ of plasma cells. These findings were consistent with a monoclonal gammopathy of undetermined significance. The clinical suspicion of angioedema was not high, because the patient's complement levels, such as $\mathrm{C} 4$ and $\mathrm{C} 1 \mathrm{q}$, were all normal.

Prevention of these crises was attempted with theophylline (400 mg/day), IVIG (2 g/ kg/day for 1 day, repeated every 4 weeks), and high-dose dexamethasone (40 mg on days $1-4,9-12$, and 17-20, repeated every 4 weeks); however, five additional episodes of hypotension occurred in the subsequent 3 months after hospitalization (Fig. 1). The last one was so severe that it required repeat fasciotomy of the right forearm. Over the next 3 months, seven episodes ensued despite VMP (bortezomib, melphalan, and prednisolone) therapy, which consisted of bortezomib $\left(1.3 \mathrm{mg} / \mathrm{m}^{2}\right)$ on days $1,8,15$, and 22 with melphalan $\left(9 \mathrm{mg} / \mathrm{m}^{2}\right)$ and prednisolone $\left(60 \mathrm{mg} / \mathrm{m}^{2}\right)$ on days $1-4$. Then, daily oral thalidomide (100 mg) was started. Surprisingly, the patient had no further episodes for 2 years, except for one episode soon after the initiation of thalidomide. Notably, his monoclonal IgG $\kappa$ protein remains clearly detectable, despite the complete disappearance of his hypotensive crises.

The serum of the patient was stored on the 50th hospital day, immediately after the onset of the 5th hypotensive crisis, to profile possible mediators responsible for the capillary leak. The second sample was serum obtained on the 204th hospital day, when the patient's hypotensive crisis had disappeared after the introduction of thalidomide. The cytokine levels in the patient's serum were measured using Quantikine enzyme-linked immunosorbent assay kits (R\&D Systems, Minneapolis, MN, USA). Our institutional review board approved the study protocol, and written informed consent was obtained from the patient.

First, we focused on VEGF, Ang-2, and CXCL10, since the possible involvement of these cytokines in SCLS has been indicated in some reports [3-6]. The results presented in Table 1 show that the concentrations of VEGF, Ang-2, and CXCL10 were all within the normal range at 


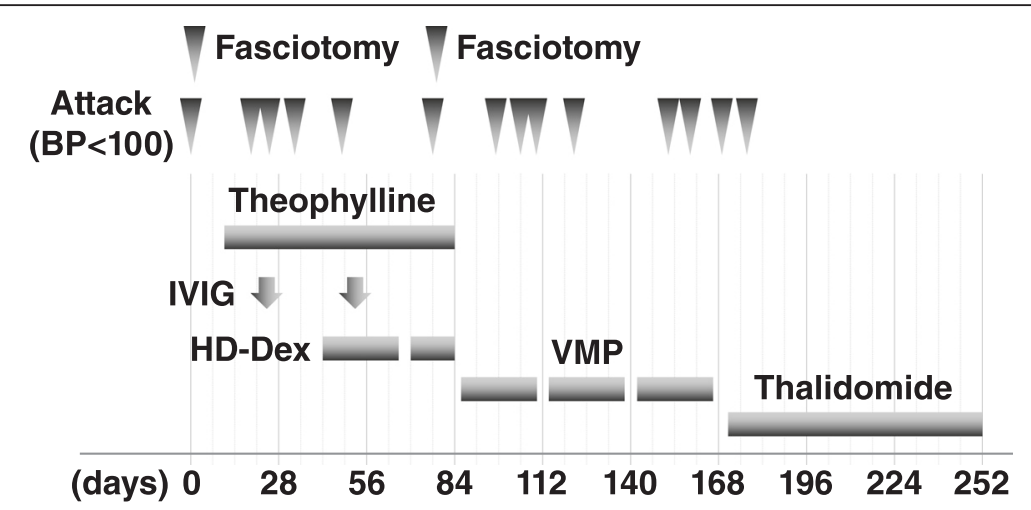

Fig. 1 Clinical course of our patient. BP blood pressure, IVIG intravenous immunoglobulin, HD-Dex high-dose dexamethasone, VMP bortezomib, melphalan, and prednisolone

each measurement point. Then, we analyzed VEGF isoforms VEGF-C and VEGF-D. The results were that the concentration of VEGF-D on the 50th day was $1042 \mathrm{ng} /$ $\mathrm{ml}$, which exceeded the normal range (135-642 $\mathrm{ng} / \mathrm{ml}$ ). In the serum obtained on the 204th day as the negative control, the VEGF-D level was normal. The concentrations of VEGF-C did not exceed the normal range on either the 50th or the 204th day. Supplemental examinations of four more cytokines-interleukin (IL)-2, IL-4, IL-6, and IL-11-were performed; however, none of them was detected.

\section{Discussion}

The etiology of SCLS has not been clarified. One possibility is a monoclonal immunoglobulin often detected in the serum of patients. The clonal plasma cells may produce a toxic monoclonal immunoglobulin that causes pathology by aggregating and depositing in tissue [9]. Although purified monoclonal immunoglobulin from one patient with SCLS failed to bind cultured endothelial cells [3], we speculate that monoclonal immunoglobulin should have an etiological function in some patients with SCLS, because sometimes IVIG is quite effective as a

Table 1 Cytokine levels in the patient's serum

\begin{tabular}{llll}
\hline & & \multicolumn{2}{l}{ Our patient } \\
\cline { 3 - 4 } Cytokines & Healthy volunteers ${ }^{\mathrm{a}}$, mean $($ range $)(n)$ & Day 50 & Day 204 \\
\hline VEGF & $220(62-707)(n=37)$ & 97 & 221 \\
Ang-2 & $2494(1065-8907)(n=60)$ & 2266 & 1899 \\
CXCL10 & $89(38-361)(n=60)$ & 251 & 312 \\
VEGF-C & $4847(2459-6651)(n=30)$ & 2133 & 4100 \\
VEGF-D & $297(135-642)(n=60)$ & 1042 & 403 \\
\hline
\end{tabular}

VEGF vascular endothelial growth factor, Ang-2 angiopoietin 2, CXCL10 C-X-C motif chemokine 10, VEGF-C vascular endothelial growth factor-C, VEGD-D vascular endothelial growth factor-D

Values are given in picograms per milliliter.

aThe data indicated in the manufacturer's written instructions prophylactic therapy for hypotensive episodes [1]. IVIG may neutralize pathogenic monoclonal immunoglobulin, which is a widely accepted theory in treatment of immune thrombocytopenic purpura. Although monoclonal immunoglobulin was clearly detected after the disappearance of the hypotensive crises by the introduction of thalidomide, the possible involvement of monoclonal immunoglobulin as an etiology in our patient could not be ruled out.

Another plausible etiology is the soluble mediators. Recently, three patients with SCLS were reported to have high levels of plasma VEGF during acute episodes, which decreased with symptom resolution [3,5]. Further, Xie et al. examined VEGF and Ang-2 in 20 patients with SCLS and found that these were elevated in episodic SCLS sera but not in remission sera [4]. Xie et al. also reported recently that CXCL10 was significantly increased at baseline and in acute SCLS sera relative to controls [6]. Meanwhile, in episodic sera of our patient, not VEGF but its D isoform, VEGF-D, was detected. VEGF-D contributes to VEGF receptor (VEGFR)-2 signaling, which is the major mediator of the mitogenic, angiogenic, and permeabilityenhancing effects of VEGF [10]. Thus, VEGF-D, instead of VEGF, may have been a causative mediator of hypotensive crises in our patient. Further, VEGF-D signals through not only VEGFR-2 but also VEFGR-3 (Flt-4). VEGF-D is expressed in the vascular endothelium. VEFGR-3 is expressed predominantly in lymphatic endothelial cells and regulates lymphangiogenesis. VEGF-D has also been implicated in lymphedema. We speculate that VEGF-D may be involved in patients with SCLS whose serum VEGF is not elevated [11] or in patients for whom antiVEGF antibody bevacizumab is not effective [3], since bevacizumab recognizes VEGF but not VEGF-D [12].

As this report is the first, to the best of our knowledge, that names VEGF-D as a causative mediator, further evaluation in more patients is necessary. Measuring VEGF-D in serum soon after the hypotensive episode 
might be suggested in some patients with SCLS whose serum VEGF, Ang-2, or CXCL-10 is not high.

As one more plausible etiology, we add the hypersensitivity of VEGFR-2. We speculate that not only VEGF concentration but also VEGFR-2 sensitivity regulates the onset of SCLS, since patients with multiple myeloma often have extremely elevated levels of serum VEGF concentration [13] without any symptoms of SCLS. Both congenital and acquired factors are able to contribute to the hypersensitivity of VEGFR-2. Genome-wide singlenucleotide polymorphism (SNP) analysis of patients with SCLS demonstrated that several SNPs were detected in $\beta$-transducin repeat containing E3 ubiquitin protein ligase and dysferlin, which could regulate VEGFR2 signaling [14]. Further examination in these areas is needed.

There remains a problem regarding how thalidomide controlled our patient's VEGF-D-induced hypotensive crises. Since thalidomide as a single agent achieves only approximately $30 \%$ of overall response, with a complete response rate probably in the $10 \%$ to at best $15 \%$ range even in patients with proliferative myeloma, the eradication of clonal plasma cells with thalidomide in our patient could not be expected. A useful reference is a report by Gupta et al., who demonstrated that thalidomide suppressed the production of VEGF from myeloma cells and bone marrow stromal cells [15]. Also, in our patient, thalidomide may have suppressed VEGF-D production from clonal plasma cells or bone marrow stromal cells.

There have been two reports presenting a total of four patients with SCLS treated effectively with thalidomide $[1,8]$. Although we have no objection to the opinion of Gousseff et al. that $\beta_{2}$-agonists and IVIG provide prophylaxis as the first-line therapy [8], there must be a certain group of patients with SCLS who could be treated effectively with thalidomide.

\section{Conclusions}

We present a case of SCLS with elevated serum VEGF-D concentration whose hypotensive crises were well-controlled with thalidomide.

\section{Consent}

Written informed consent was obtained from the patient for publication of this case report and any accompanying images. A copy of the written consent is available for review by the Editor-in-Chief of this journal.

\section{Authors' contributions}

SIb and TS drafted the manuscript. AT, AH, YK, JW, HH, and $\mathrm{KO}$ were involved in the care of the patient. KT, KMu, and Sly analyzed and interpreted the patient data. YS, KMi, MK, RT, and JK revised the manuscript for important intellectual content. All authors read and approved the final manuscript.

\section{Competing interests}

The authors declare that they have no competing interests.

Received: 16 February 2015 Accepted: 7 April 2016

Published online: 24 May 2016

\section{References}

1. Gousseff M, Arnaud L, Lambert M, Hot A, Hamidou M, Duhaut P, et al. The systemic capillary leak syndrome: a case series of 28 patients from a European registry. Ann Intern Med. 2011;154:464-71.

2. Kapoor P, Greipp PT, Schaefer EW, Mandrekar SJ, Kamal AH, Gonzalez-Paz NC, et al. Idiopathic systemic capillary leak syndrome (Clarkson's disease): the Mayo clinic experience. Mayo Clin Proc. 2010;85:905-12.

3. Lesterhuis WJ, Rennings AJ, Leenders WP, Nooteboom A, Punt CJ, Sweep FC, et al. Vascular endothelial growth factor in systemic capillary leak syndrome. Am J Med. 2009;122:e5-7.

4. Xie Z, Ghosh CC, Patel R, Iwaki S, Gaskins D, Nelson C, et al. Vascular endothelial hyperpermeability induces the clinical symptoms of Clarkson disease (the systemic capillary leak syndrome). Blood. 2012;119:4321-32.

5. Kinoshita Y, Kasaoka S, Fujita M, Oshima C, Kawamura Y, Tsuruta R, et al. Synchronized changes in serum vascular endothelial growth factor during the clinical course of chronic systemic capillary leak syndrome. Intern Med. 2010;49:791-4

6. Xie Z, Chan E, Yin Y, Ghosh CC, Wisch L, Nelson C, et al. Inflammatory markers of the systemic capillary leak syndrome (Clarkson disease). J Clin Cell Immunol. 2014;5:1000213.

7. Yabe H, Yabe M, Koike T, Shimizu T, Morimoto T, Kato S. Rapid improvement of life-threatening capillary leak syndrome after stem cell transplantation by bevacizumab. Blood. 2010;115:2723-4.

8. Staak JO, Glossmann JP, Esser JM, Diehl V, Mietz H, Josting A. Thalidomide for systemic capillary leak syndrome. Am J Med. 2003;115:332-4.

9. Druey KM, Greipp PR. Narrative review: the systemic capillary leak syndrome. Ann Intern Med. 2010:153:90-8.

10. Clarke JM, Hurwitz HI. Understanding and targeting resistance to antiangiogenic therapies. J Gastrointest Oncol. 2013;4:253-63.

11. Nagao $Y$, Harada H, Yamanaka H, Fukuda K. Possible mediators for systemic capillary leak syndrome. Am J Med. 2011;124:e7-9.

12. $Y u Y$, Lee $P$, Ke $Y$, Zhang $Y, Y u$ Q, Lee J, et al. A humanized anti-VEGF rabbit monoclonal antibody inhibits angiogenesis and blocks tumor growth in xenograft models. PLoS One. 2010;5:e9072.

13. Iwasaki T, Hamano T, Ogata A, Hashimoto N, Kitano M, Kakishita E. Clinical significance of vascular endothelial growth factor and hepatocyte growth factor in multiple myeloma. Br J Haematol. 2002;116:796-802.

14. Xie Z, Nagarajan V, Sturdevant DE, Iwaki S, Chan E, Wisch L, et al. Genomewide SNP analysis of the systemic capillary leak syndrome (Clarkson disease). Rare Dis. 2013;1:e27445.

15. Gupta D, Treon SP, Shima Y, Hideshima T, Podar K, Tai YT, et al. Adherence of multiple myeloma cells to bone marrow stromal cells upregulates vascular endothelial growth factor secretion: therapeutic applications. Leukemia. 2001;15:1950-61.

Submit your next manuscript to BioMed Central and we will help you at every step:

- We accept pre-submission inquiries

- Our selector tool helps you to find the most relevant journal

- We provide round the clock customer support

- Convenient online submission

- Thorough peer review

- Inclusion in PubMed and all major indexing services

- Maximum visibility for your research 